Guy Brucy, Françoise F. Laot, Emmanuel de Lescure (dir.), Former les militants, former les travailleurs. Les syndicats et la formation depuis la Seconde Guerre mondiale

Paris, L'Harmattan, coll. « Histoire et mémoire de la formation », 2015

\title{
Nicolas Simonpoli
}

\section{OpenEdition}

Journals

\section{Édition électronique}

URL : http://journals.openedition.org/travailemploi/7192

DOI : 10.4000/travailemploi.7192

ISSN : 1775-416X

Éditeur

DARES - Ministère du Travail

\section{Édition imprimée}

Date de publication : 1 juillet 2016

Pagination : 129-132

ISSN : 0224-4365

\section{Référence électronique}

Nicolas Simonpoli, « Guy Brucy, Françoise F. Laot, Emmanuel de Lescure (dir.), Former les militants,

former les travailleurs. Les syndicats et la formation depuis la Seconde Guerre mondiale », Travail et Emploi [En ligne], 147 | juillet-septembre 2016, mis en ligne le 11 juillet 2019, consulté le 25 septembre 2020. URL : http://journals.openedition.org/travailemploi/7192 ; DOI : https://doi.org/10.4000/travailemploi 7192 


\title{
Former les militants, former les travailleurs. Les syndicats et la formation depuis la Seconde Guerre mondiale
}

\author{
Guy Brucy, Françoise F. Laot., Emmanuel de Lescure (dir.)
}

Paris, L'Harmattan, coll. « Histoire et mémoire de la formation », 2015, 222 p.

Lu par Nicolas Simonpoli*

En janvier 2008 se tenait à Amiens un colloque sur les relations entre « syndicalisme et formations ». Cette manifestation donna lieu à une première publication parue un an plus tard sous le titre Mouvement ouvrier et formation. Genèses. De la fin du XIX siècle à l'après Seconde Guerre mondiale (L'Harmattan, 2009). Organisée à l'initiative du Groupe d'étude Histoire de la formation des adultes (Gehfa) et placée sous la direction de Guy Brucy, de Françoise F. Laot et d'Emmanuel de Lescure, cette rencontre mobilisa des sociologues et des historiens spécialistes de la formation et de l'éducation. Le présent ouvrage constitue le second volume tiré de ce colloque. Il regroupe diverses contributions dédiées aux évolutions de la formation syndicale de la Libération à nos jours. Hasard du calendrier scientifique, sa parution intervient un an après celle de l'importante synthèse de Nathalie Ethuin et de Karel Yon, La Fabrique $d u$ sens syndical ${ }^{1}$, elle aussi consacrée à cette thématique.

La première partie de l'ouvrage revient sur le passage de l' « éducation ouvrière » à la « formation syndicale ». À l'origine constituée en opposition au système scolaire considéré comme «bourgeois », la formation des militants du mouvement ouvrier s'est par la suite rapprochée des institutions de l'État. L'autonomie des écoles syndicales et politiques s'est progressivement estompée au profit d'une « reconnaissance » et d'une légitimation par le législateur. Un tournant historique s'effectue au milieu des années 1950 avec la création des Instituts du travail (1955) et l'instauration du congé d'éducation ouvrière ouvert à tous les salariés (1957). Viendront ensuite la loi de 1971 sur la formation professionnelle puis celle de 1985 sur «le congé de formation économique, sociale et syndicale », autant de réformes qui « montrent bien le poids [grandissant] de l'État en matière de formation syndicale » (p. 15). Au cours des années 1980-1990, la professionnalisation de l'activité de formation au sein des confédérations, ainsi que l'augmentation de la dotation financière publique attribuée

\footnotetext{
* Institut des sciences sociales du politique (ISP), université Paris Nanterre.

1. Ethuin N., Yon K. (2014), La Fabrique du sens syndical. La formation des représentants des salariés en France (1945-2010), Bellecombe-en-Bauges, Éditions du Croquant.
} 
aux dispositifs de formation syndicale participent à la consolidation de ce processus d'hétéronomisation. Cette dynamique est symbolisée par les Instituts du travail étudiés par Lucie Tanguy. Mêlant universitaires, éducateurs syndicaux et syndicalistes, ils ont pour vocation originelle d'associer l' « expérience du monde ouvrier et les acquis de la culture bourgeoise » (p. 28). Ils illustrent les prémisses d'une « ouverture » de la formation syndicale. La pédagogie utilisée est innovante, inspirée par les méthodes de la formation pour adulte, l'apprentissage y est collectif et valorise le savoir de chacun, l'enseignement s'effectue dans un lieu nouveau, l'université. Néanmoins, la conciliation des approches universitaire et syndicale relève aussi de l'injonction contradictoire et l' « enchantement » des débuts laisse progressivement place à une relation « utilitaire » marquée par la difficile retraduction de l' « universel » académique dans la demande de savoirs syndicaux « concrets ».

La relation entre ces deux types de production - syndicale et scientifique - est justement questionnée dans la contribution d'André Robert portant sur les « tremblés épistémologiques » de l'apport syndical dans la recherche en éducation (p. 48). L'auteur formule l'hypothèse d'une ouverture du monde scientifique aux contributions externes permettant une circulation des idées savantes dans des « zones frontalières » au sein desquelles s'effectuerait une rencontre entre connaissances scientifique et militante. Cette démonstration apparaît tout à fait pertinente dans la réaffirmation d'une différence entre productions syndicales et académiques ; toutefois on peut regretter qu'elle ne soit pas repositionnée dans un contexte historique précis, et qu'elle n'explicite pas les données empiriques sur lesquelles elle se fonde.

La deuxième partie, intitulée « formation des militants et stratégies syndicales », s'intéresse aux multiples usages de la formation. Les auteurs tentent de comprendre ce que cette «éducation » des militants nous dit du syndicalisme en interrogeant tout d'abord les contenus dispensés aux syndiqués. Leur observation confirme une logique de technicisation de l'activité syndicale qui se manifeste par une bipolarisation de la formation avec, d'une part, des enseignements d'ordre « politique » intégrant des savoirs - historiques, philosophiques, critiques, etc. - hérités du mouvement ouvrier et, d'autre part, une formation technique attachée à l'inculcation de savoir-faire juridiques nécessaires à l'exercice des mandats de représentant des salariés. La distinction, idéaletypique, entre ces deux registres laisse voir les ambitions multiples - et parfois contradictoires - poursuivies par les dispositifs de formation, si bien que des conflits internes aux écoles syndicales émergent parfois, notamment lorsque les aspirations des stagiaires entrent en contradiction avec celles des formateurs. Comme l'explique Jean-Robin Merlin à propos du syndicat SUD-PTT, les éducateurs valorisent des connaissances " généralistes » offrant aux adhérents la possibilité de comprendre les orientations de l'organisation. Or, à l'inverse, les militants « de base » privilégient des apprentissages « immédiatement utilisables » plus à même de les aider à retrouver une assurance juridique indispensable dans le cadre de leurs fonctions au sein de l'entreprise. La formation est vécue comme un moyen de mieux endosser le rôle de représentant dans la mesure où elle confirme - et assigne - aux élus leur identité de syndicaliste. 
Les auteurs reviennent également sur les appropriations - individuelles ou collectives - qui sont faites des enseignements. À ce titre, le chapitre que Nathalie Ponsart consacre à l' « incitation à la lecture » est particulièrement fécond. Entre 1945 et 1970, cette prescription apparaît comme l'un des invariants des programmes de formation syndicale. Considérée à la fois comme une « arme idéologique » et comme un outil du développement culturel du militant, la pratique de la lecture se trouve directement reliée à l'émancipation de la classe ouvrière. Être cultivé, être informé relève « [du] devoir », « [de] la nécessité » pour celui ou celle qui veut s'approprier l' « esprit critique » de l'organisation (p. 69). Les témoignages recueillis par l'auteure sont édifiants et démontrent toute l'importance de la formation militante dans la trajectoire culturelle de syndicalistes au parcours scolaire interrompu prématurément. In fine, elle évoque les conjonctures particulières dans lesquelles les incitations à la lecture critique se sont retournées contre l'institution. «Par l'émancipation revendiquée et appropriée par les militants, les lectures normatives se muent alors en lectures subversives » (p. 77) et encouragent une prise de distance avec les orientations du syndicat.

Dans un troisième temps ce sont les modalités de l'apprentissage qui sont interrogées. Si au travers de réseaux d'éducateurs, l'institution met avant tout en œuvre une formation aux caractéristiques « scolaires », les voies d'éducation détournées n'en sont pas moins importantes. Ainsi, la transmission de savoir-faire « par capillarité » ou « sur le tas » demeure fréquente (p. 81). Ces modes de diffusion traditionnels de la culture syndicale sont particulièrement prégnants dans l'analyse prosopographique proposée par Stéphane Paquelin. Son approche « par les parcours militants » démontre tout l'intérêt d'inscrire les trajectoires biographiques dans des contextes géographiques et générationnels précis. En effet, suivant les lieux et les périodes, l'insuffisance de l'offre de formation contraint parfois les syndicalistes à développer des savoirs « autodidactes » construits avec les sociabilités militantes, au cours des mouvements de luttes, etc., qui ne sont sanctionnés qu' a posteriori par l'organisation. Toutefois, si les analyses de l'auteur apparaissent tout à fait convaincantes, on peut regretter que les transformations du système de formation initiale depuis la Libération ne soient pas mises en lien avec la fréquentation des écoles syndicales.

Enfin, la dernière partie du livre se concentre sur les rapports entre formations syndicale et professionnelle. À l'origine distinctes, ces deux branches tendent à être rapprochées et englobées sous la notion de «formation permanente». Un tournant a été opéré au début des années 1970 lorsque « le syndicalisme intègre dans son approche revendicative la nécessité d'une formation professionnelle continue » (p. 148). Depuis, cet enjeu fait l'objet d'une sorte de consensus entre représentants de l'État, du patronat et des salariés. Néanmoins si tous les acteurs s'accordent sur le principe d'un plus large accès des salariés et des représentants syndicaux à la formation - notamment universitaire et professionnelle -, des divergences perdurent sur les moyens à mettre en œuvre pour y arriver. Ainsi, du côté syndical, deux registres de légitimation s'opposent avec, d'un côté, «l'altérité ouvrière » défendant l'autonomie du groupe ouvrier et, de l'autre, « la filiation républicaine » encourageant l'émancipation individuelle 
et l'intégration culturelle. À ce titre, comme l'explique Louis-Marie Barnier, les dispositifs de validation des acquis de l'expérience (VAE) «militante », très utilisés par certaines universités françaises, sont de parfaits exemples des contradictions inhérentes au passage d'une posture " autonome » à une posture « intégrée ». La reconnaissance universitaire de l'activité syndicale se heurte en effet à certaines limites : la catégorie de « militant » est-elle pertinente au vu de son hétérogénéité ? Comment des savoir-faire politiques, voire subversifs, peuvent-ils être retraduits sous la forme de « compétences professionnelles »? Dans quelle mesure des activités syndicales exercées collectivement peuvent-elles être appropriées individuellement dans le cadre d'une VAE ? Autant d'éléments analysés dans les deux derniers chapitres de ce volume ${ }^{2}$. De ceux-ci nous retiendrons l'idée qu'en légitimant des mécanismes individuels de certification, les confédérations s'inscrivent en rupture avec une tradition syndicale attachée aux normes collectives et impersonnelles. Aussi, on peut interroger avec Fabienne Maillard le processus de «conversion» (p. 212) entrepris par les organisations syndicales, les évolutions successives de la formation pouvant dès lors également révéler les transformations générales touchant les centrales françaises.

On l'aura compris, ces différentes contributions offrent un panorama d'approches scientifiques sur la formation syndicale. L'ensemble constitue un outil très utile pour tout chercheur travaillant sur le syndicalisme. Néanmoins, le lecteur regrettera que ne soient pas étudiées certaines évolutions récentes de l'objet, notamment l'émergence d'un marché privé de la formation des institutions représentatives du personnel (IRP) venant concurrencer les entreprises syndicales traditionnelles. De plus, on constate une tendance des auteurs à autonomiser la formation du reste de l'activité syndicale. Or, un décentrement du regard - qui serait alors particulièrement axé sur les trajectoires biographiques - permettrait de mesurer la réelle influence de la formation sur le corps militant mais aussi d'interroger plus largement les liens entre cursus syndical, scolaire, partisan, professionnel, etc.

2. Sur le même thème, voir également Neyrat F. (2006), La Validation des acquis de l'expérience. La reconnaissance d'un nouveau droit, Bellecombe-en-Bauges, Éditions du Croquant. 